\title{
Homogenization of Polymeric Flocculant Solutions Used for Purification of Slurry Waters After Coal Preparation
}

\author{
Galina Evmenova ${ }^{1, *}$ and Sergey Evmenov ${ }^{2}$ \\ ${ }^{1}$ T.F. Gorbachev Kuzbass State Technical University, Department of Mineral Processing, \\ 650000, Kemerovo, Russian Federation \\ ${ }^{2}$ T.F. Gorbachev Kuzbass State Technical University, Department of Coal Chemistry, Plastics \\ Materials and Environmental Engineering, 650000, Kemerovo, Russian Federation
}

\begin{abstract}
Use of flocculants - high-molecular synthetic water-soluble polymers, is one of the most perspective and economical methods for purification of the return and waste waters from coal preparation plants, and intensification of the whole coal processing process. For efficient use of flocculants, it is necessary to prepare homogeneous aqueous solutions, for which an optimal mixing mode should be selected, taking into account energy costs and quality of the concentrated solution. The paper presents herein result of the homogenization process analysis at preparation of solutions used for slurry water purification. Based on study of the rheological solution behavior and determination of the required for the numerical analysis of the process parameters of the rheological constants, power costs, homogenization time, type of equipment and mixing device have been determined.
\end{abstract}

\section{Introduction}

Currently, Kuzbass receives a great number of foreign-made flocculants different in their molecular weight, structure, mechanism of action, ionic strength and other characteristics that should be considered while using them. Effectiveness of flocculants depends on the molecular weight and polymer structure, charge of functional groups, as well as properties of the flocculated particle surface, and method of solution preparation, etc. These factors should provide active adsorption at forming units and affect the flow rate of flocculants [14].

Besides, earlier studies have shown [5-8] that using flocculants, preparation of their aqueous solutions, since this is the only way to disperse water-soluble polymers to the molecular level, to change the conformation of macromolecules and give them a form that promotes vigorous aggregation of the dispersed systems is important.

Dissolution process of the high-molecular polymers consists of three stages: wetting of the dispersed flocculant particles with water forming a hydrate layer on the particles surface; swelling characterizing by diffuse penetration of water molecules into the intermolecular space of the polymer to form a gel; directly dissolving a polymer in water, i.e. the transition of the flocculent macromolecules into the solvent volume.

* Corresponding author: galina-evmenova@yandex.ru 
Comprehensive studies of the wetting processes, dissolution and destruction of various types of flocculants have shown the requirement to develop a technology for solutions preparation in a 'gentle' mode and appropriate hardware design [9-11].

\section{Materials \& Methods}

To implement technology of preparation of flocculant working solutions, it is necessary to establish a homogenization (mixing) mode in terms of optimal energy parameters and mixing quality at the stage of the concentrated solution obtaining. The most expedient way is to carry out the process in standard equipment with agitators, the dimensions of which depend on the required solution volume. The main parameters of the process are the power consumed during homogenization process, and the time to achieve a specific technological result, i.e. the time of homogenization.

Determination of these parameters depends on the hydrodynamic pattern created in the unit during mixing, as well as the rheological behavior and rheological properties of the working medium [12]. It should be noted that the rheological behavior of the polymer system depends on a number of factors, including molecular weight, molecular weight distribution, polymer concentration, and solvent viscosity etc.

In this regard, the authors conducted a separate series of studies on the rheological behavior of the flocculant solutions in the weight concentration range of $0.1 \ldots 0.5 \%$. Object of study were flocculants based on polyacrylimide of Magnafloc brands 525, 365 and 1440 (M 525, M 365 and M 1440), as well as polyethylene oxide (PEO).

The experimental study of the polymer flocculant solutions viscosity has been carried out on rotary viscometer RheotesT-2 of the 'cylinder-cylinder' type (made by RheoTest Messgerate Medingen $\mathrm{GmbH}$, Germany), variation range of shear rates $Y$ was $5,4 \ldots 1312 \mathrm{c}^{-1}$ and fully covered possible limits of the intensity of deformation effects on working medium while solutions preparation. The determining method of the shear rate for this type of devices has been described in the work [13].

For the flocculant solutions under study, also the dependence curve of viscosity $\eta$ to concentration $\eta(c)$, shear rate $\eta(\dot{Y})$, and flows that are the dependence of the shear stress $\tau$ to shear rate $\tau(\dot{Y})$ have been obtained. It has been figured out that the rheological properties of the solutions are greatly influenced by concentration of polymer $c$ and shear rate $\dot{Y}$. Dependences $\eta(\dot{Y})$ and flow curves for all Magnaflock brands characterize solutions of these flocculants as non-Newtonian fluids, the properties of which are described by the known Ostwald - De Villa power equation [14-16]:

$$
\tau=k \cdot \dot{Y}^{m}
$$

Constant $k$ depends on the medium consistency, and value $m$ is called 'flow index' and shows how the rheological behavior of the liquid differs from the Newtonian. NonNewtonian fluids for which $m<1.0$, are called pseudo-plastic, moreover, the smaller the flow index value is, the more rheological behavior of fluid differs from the Newtonian one. Constants $k$ and $m$ are determined from the flow curve $\tau(\dot{Y})$ for all solutions under study (Table. 1).

Solutions of polyethylene oxide before $c=0.25 \%$ exhibit Newtonian properties, and viscosity anomaly begins to appear only at the flocculant concentration of $0.5 \%$ or greater $\left(k \cdot 10^{-2}\right.$ varies from 1.8 to $11.3 ; m$ - from 0.858 to 0.831$)$.

To implement technology of flocculant working solutions preparation, it is essential to establish a homogenization (mixing) mode in terms of optimal energy parameters and mixing quality at the stage of the concentrated solution obtaining. It is expedient to carry 
out this process in standard units with agitators, dimensions of which depend on the required volume of the solution.

Table 1. Constants of power rheological equation for flocculant solutions.

\begin{tabular}{|c|c|c|c|c|c|c|}
\hline \multirow{2}{*}{$\begin{array}{c}\text { Solution } \\
\text { concentration } \boldsymbol{c}, \boldsymbol{\%}\end{array}$} & \multicolumn{5}{|c|}{ Flocculant name } \\
\cline { 2 - 7 } & \multicolumn{2}{|c|}{ M 365 } & \multicolumn{2}{|c|}{ M 525 } & \multicolumn{2}{c|}{ M 1440 } \\
\cline { 2 - 7 } & $\boldsymbol{k}$ & $\boldsymbol{m}$ & $\boldsymbol{k}$ & $\boldsymbol{m}$ & $\boldsymbol{k}$ & $\boldsymbol{m}$ \\
\hline 0.10 & 0.273 & 0.468 & 0.368 & 0.452 & 0.205 & 0.498 \\
\hline 0.15 & 0.511 & 0.455 & 1.031 & 0.41 & 0.443 & 0.453 \\
\hline 0.25 & 0.849 & 0.433 & 1.319 & 0.40 & 0.709 & 0.441 \\
\hline 0.50 & 2.521 & 0.365 & 1.849 & 0.377 & 1.306 & 0.403 \\
\hline
\end{tabular}

It is known [12] that for such devices power consumed during the mixing is included into the expression of a so-called power criterion $K_{N}$ :

$$
K_{N}=\frac{N}{\rho \cdot n^{3} \cdot d_{M}^{5}}
$$

where $N$ - consumed power; $\rho$ - solution density; $n$ - agitator rotation velocity; $d_{M}$ - agitator diameter.

Criterion equation of power costs in geometrically similar devices with agitators can be expressed in a form of power monomial:

$$
K_{N}=C \cdot\left(\operatorname{Re}_{c}\right)^{\alpha},
$$

where $C$ and $\alpha$-experimentally obtained coefficients.

For the correct use of the study results of the flocculant solutions rheological properties, it is important to determine a range of the actual shear rates applied in the technological process, since the hydrodynamics of non-Newtonian fluid flows in the units with agitator greatly depends on the rheological behavior of the deformable medium.

In particular, it has been found [17] that for the pseudo-plastic media shear rate $\dot{Y}$, with increasing distance from the agitator axis, decrease faster than for Newtonian ones, and zone of the intensive action of the agitator narrows. Maximum value $Y$ is achieved in the area directly adjacent to the surface of the agitator blades at radius approximately equal to the agitator radius. As per some authors, [12] the following approximate dependence can be used to determine shear rate in this area:

$$
\dot{Y}=A \cdot n
$$

where $A$ - characteristic value for corresponding type of device (in particular, for turbine, blade and propeller devices at $D / d_{M} \geq 1,5$ value $\left.A=11\right) ; D$-device diameter.

The Reynolds centrifugal criterion $R e_{c}$ that considers peculiarities of the rheological behavior of the medium, as mentioned hereinabove, makes it possible to take into account the non-Newtonian behavior of the working medium in the immediate vicinity of the agitator and is computed by the following expression:

$$
R \mathrm{e}_{c}=\frac{\rho \cdot n^{2-m} \cdot d_{\mathrm{M}}^{2}}{k}(A)^{1-m},
$$

where $k$ and $m$ - constants of the Ostwald-De Villa rheological power equation (1). 
Testing of modes for the flocculants solutions obtaining has been carried out under production conditions using industrial unit equipped with a blade agitator of the following dimensions: unit diameter $D=1.8 \mathrm{~m}$, agitator diameter $d_{\mu}=0,63 \mathrm{~m}$, ratio $D / d_{M}=2.86$. Variation range of agitator rate of rotation has been from 1.0 to $130 \mathrm{rpm}\left(0.017-2.17 \mathrm{c}^{-1}\right)$.

Shear rate $\dot{Y}$ determined by equation (4) at a maximum agitator rate of rotation $2.17 \mathrm{c}^{-1}$ is $23.87 \mathrm{c}^{-1}$, and is within the range of values $Y$ at which constants of the power rheological equation $k$ and $m$ have been determined (table 1). Knowing the numerical values of these constants, as well as considering that density of the studied solutions has a value of about $1000 \mathrm{~kg} / \mathrm{m}^{3}$, it is possible to determine $R e_{c}$ values at changing agitator rate of rotation from minimum $0.017 \mathrm{c}^{-1}$ to maximum value $2.17 \mathrm{c}^{-1}$. Total variation range in value $R e_{c}$ for all the studied solutions is $1.0 \ldots 2.2 \cdot 10^{4}$. To determine power criterion $K_{N}$, values of coefficients $C$ and $\alpha$ are taken from the criterion equation (3), which are given in the paper [12], and for the blade agitator we obtain the dependence $K_{N}\left(R e_{c}\right)$, presented on Fig.1 (curve 1).

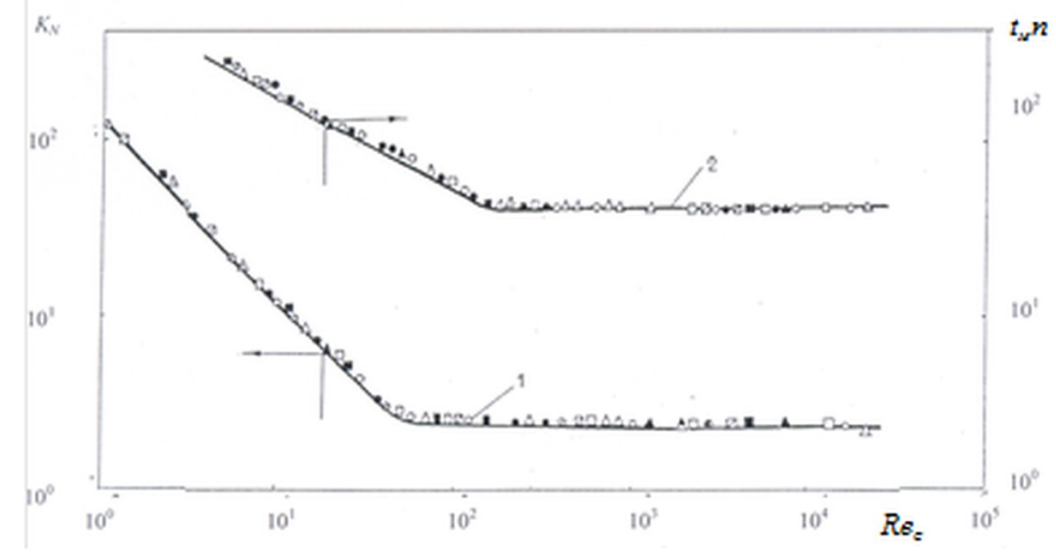

Fig. 1. Dependence of capacity criterion $K_{N}$ (curve 1) to homochronicity $t_{M} \cdot n$ (curve 2) to $\operatorname{Re}_{c}$.

The peculiarity of this dependence is that values $K_{N}$ for all concentrations of the studied solutions fit well on one curve. The first section of this curve is characterized by a drop in value $K_{N}$, covers almost two decimal exponent of the Reynolds centrifugal criterion and is ended at $R e_{c}$ approximately equal to 90 .

The second section of this dependence is characterized by such a small decrease in the power criterion that it can be neglected and without much error to assume that in this section $K_{N}=$ const. For the number $R e_{c} \geq 90$ that corresponds to the agitator rate of rotation $5 \mathrm{rpm}$, the power consumed for mixing is almost constant. This is well explained by the non-Newtonian behavior of the flocculant solutions at flow, when an increase in deformation rate leads to a decrease in viscosity and reduces resistance of the medium during deformation.

Therefore, from the power cost minimizing point of view in the process of the solutions preparation applying minimum agitator rate of rotation of diameter $d_{\mathrm{m}}$, as mentioned hereinabove, has been a value of $5 \mathrm{rpm}\left(0.083 \mathrm{~s}^{-1}\right)$.

From the presented dependence it follows that in case of technological necessity the agitator rate of rotation can be increased within entire studied range of revolutions, a maximum value of which in this case is $130 \mathrm{rpm}$ without increasing power criterion.

A mixing medium homogenization time $t_{M}$ is almost completely determined by the hydrodynamic pattern in the unit and can be considered as duration of the hydrodynamic stabilization of the flocculant solution flow, driven from a state of rest. Value $t_{\mu}$ is included 
into a so-called criterion of homochronicity, which applied to the mixing process, is represented by $t_{\mu} \cdot n$, and is determined by the following criterion equation:

$$
t_{M} \cdot n=C_{l}\left(\operatorname{Re}_{c}\right)^{\alpha}{ }_{1}
$$

Coefficient values $C_{1}$ and $\alpha_{1}$ for the blade agitator are given in the paper [12]. In the considered hereinabove range $R e_{c}$, homochonicity values $t_{\mathrm{M}} \cdot n$ have been calculated and dependency $t_{\mathrm{M}} \cdot n\left(R e_{c}\right)$ has been built, the type of which is almost the same as for the dependency of $K_{N}\left(R e_{c}\right)$ (Fig. 1, curve 2). Minimum homogenization time corresponds to value $R e_{u}=2 \cdot 10^{2}(n=10 \mathrm{rpm})$ and is about 15 minutes. With further increase of $n$, the computed value $t_{\mathrm{M}}$ does not change

As it noted hereinabove, while mixing of the pseudo-plastic media described by the power rheological equation (1), to which the studied solutions of flocculants are referred, shear rate with increasing distance from the agitator axis falls faster versus to Newtonian ones. It should be taken into account implementing technological process, since it can reduce the effectiveness of the agitator mixing effect. Moreover, with a decrease in index of flow $m$, this peculiarity of the process appears brighter [17]. Magnaflock M 365 index of flow, at increasing solution concentration from 0.1 to $0.5 \%$ decreases on $22.2 \%$ (Table), M 525 - on $16.6 \%$, and M 1440 - on $19 \%$.

Therefore, it becomes obvious that for more concentrated Magnaflock solutions it is essential to increase hydrodynamic effect during mixing, for example, by increasing agitator rate of rotation. The limit value of rotation velocity will be the value at which mechanical destruction of the flocculant macromolecules in solution begins to appear actively.

\section{Conclusions}

It has been experimentally established that based on study of the rheological properties of the flocculant solutions you can select:

- type of unit and mixing device;

- determine agitator speed and energy costs;

- determine optimal mixing time to obtain a homogenized solution of the polymer flocculants.

\section{References}

1. R. Nitzsche, M. Muller, Chem. Ind., 6, 10 (1992)

2. K. Balasubramani, R. G. Bhaskar, Indian Mining and Eng. J., 7, 7 (1990)

3. Z. Wagberg, T. Zindstrom, L. Morgan, Powder Technol, 2, 77 (1988)

4. S. Smith, G. Walles, Chem. Process (USA), 4, 80 (1992)

5. B. Vincent, Adv. Colloid Interface Sci., 4, 196 (1974)

6. V. K. La Mer, Disc.Faraday Soc., 42, 248 (1966)

7. W. E. Walles J. Colloid Interface Sci., 27, 797 (1968)

8. R. H. Smellie, V.K. La Mer J. Colloid Shi., 6, 589 (1959)

9. G. L. Evmenova, A. A. Baichenko, J. Min. Sci., 36, 518 (2000)

10. M .H Al-Rashed, M. Lemanowicz, A.T. Gierczycki, Int. J. Miner. Process., 104:105, 1 (2012) 
11. S. D. Evmenov, G.L. Evmenova, Chinese Coal in the XXI Century, Mining, Green and Safety Taishan Academic Forum - Project on Mine Disaster Prevention and Control, 424, 107 (2014)

12. Z. Sterbacek, P. Tausk, Michani v chemickem prumslu (Statni nakladatelstvi technicke literatury, Praha, 1959)

13. I. M. Krieger, H. Elrod, J. Appl. Phys., 24, 134, (1953)

14. De Waele, Oil Color Chem. Ass. J., 6, 23, (1923)

15. Ostwald W. Kolloid-Z., 36. 99, (1925)

16. W. Ostwald, R. Averbach. Kolloid-Z., 38, 261, (1926)

17. M. Zlokarnik, Ibid., 9, 539 (1967) 